\title{
Patterns of Total Extracellular Protein Secretion by a Number of Clinically Isolated Strains of Staphylococcus aureus
}

\author{
By GEOFFREY COLEMAN, CLIFFORD M. JAKEMAN AND \\ NICHOLAS MARTIN \\ Department of Biochemistry, University Hospital and Medical School, \\ Clifton Boulevard, Nottingham NG7 $2 U H$
}

(Received 10 March 1978)

\section{INTRODUCTION}

Abbas-Ali \& Coleman (1977) studied the characteristics of total extracellular protein secretion by Staphylococcus aureus strain Wood 46. The differential rate of exoprotein secretion was low during the exponential growth phase but increased on entry into the postexponential phase of the growth cycle. Subsequently, it was demonstrated that an increase in the rate of exponential growth produced a corresponding reduction in the differential rate of exoprotein formation (Coleman \& Abbas-Ali, 1977). These characteristics were consistent with the 'competition' model for the regulation of extracellular protein formation described by Coleman, Brown \& Stormonth (1975).

It is possible that the characteristics of the highly haemolytic Wood 46 strain of S.aureus, which has been widely used in laboratory studies, differed from clinically isolated strains of the organism. This possibility has been examined by taking a random selection of clinical isolates and determining their patterns of total exoprotein production under conditions similar to those used by Abbas-Ali \& Coleman (1977).

\section{METHODS}

Organisms. Ten recently isolated strains of Staphylococcus aureus (numbered 1 to 10) were kindly provided by the Public Health Laboratories, Hucknall Road, Nottingham. These strains were compared with the Wood 46 strain previously studied by Abbas-Ali \& Coleman (1977).

Growth conditions. Overnight cultures were grown in a $3 \%(\mathrm{w} / \mathrm{v})$ Tryptone Soya Broth medium supplemented with vitamins and metal ions as described by Abbas-Ali \& Coleman (1977). Further batches of medium $(500 \mathrm{ml}$ in 21 conical flasks) were inoculated with $1.0 \mathrm{ml}$ samples of the overnight cultures and incubated at $37^{\circ} \mathrm{C}$ with shaking, as before. Samples of these cultures were taken at regular intervals for bacterial density and extracellular protein assays.

Bacterial dry weight determination. The method of Stormonth \& Coleman (1972) was employed.

Extracellular protein estimation. The protein contents of samples of culture supernatant fractions, which had been diluted 15 -fold with water, were determined by the Coomassie brilliant blue method of Sedmak \& Grossberg (1977). In order to relate these results to those reported previously, the last sample for each progress curve was assayed by the modified biuret method of Bürgi, Richterich \& Briner (1967), with bovine serum albumin (Sigma) as a standard. These latter values together with the corresponding spectrophotometer data obtained by the Coomassie brilliant blue method were used to determine the specific absorption coefficient in each case.

Haemolytic activity. The maximum haemolytic activity achieved in each culture was determined as described by Abbas-Ali \& Coleman (1977). 


\section{RESULTS}

The 10 clinical isolates studied were obtained from a wide variety of sources and had very different phage typing patterns. The maximum bacterial densities which these organisms reached during a $23 \mathrm{~h}$ incubation period covered a twofold range; the Wood 46 strain achieved an intermediate density. The abilities of the clinically isolated strains to produce exoprotein ranged from one which was similar to the Wood 46 strain to one which formed an amount of exoprotein equal to only $7 \%$ of the highest level observed. This low exoproteinproducing organism was that which achieved the lowest bacterial density in the Tryptone Soya Broth medium. Determination of the maximum haemolytic activity of each strain, using rabbit erythrocytes, showed a random distribution of low values for the clinical isolates, the highest activity recorded being only $2 \%$ of that reached by the highly haemolytic Wood 46 strain.

A comparison was made of the progress of secretion of exoprotein in relation to increase in bacterial density throughout the growth cycle by the 10 clinical isolates and the Wood 46 strain. The gross characteristics of clinical isolate no. 7 and the Wood 46 strain were similar, with low rates of exoprotein production during exponential growth which increased in the post-exponential phase of the growth cycle (Fig. 1). Clinical isolate no. 7 grew to a higher bacterial density than Wood 46 with an accompanying lesser production of exoprotein, a pattern which was not adhered to by all the other strains examined. However, the growth curves and exoprotein secretion curves of clinically isolated strains no. 1 to no. 6 were all within the bounds of the corresponding curves for Wood 46 and clinical isolate no. 7 . Further, for each strain, the rate of exoprotein secretion increased after the end of exponential growth, resulting in a biphasic differential rate of exoprotein formation, as shown in Fig. 2 for the Wood 46 strain and clinical isolate no. 7 .

The three lowest exoprotein producers did not fall within the limits set by the Wood 46 strain and clinical isolate no. 7 shown in Fig. 1. However, clinical isolate no. 8 exhibited a pattern for exoprotein formation parallel to but with lower values than no. 7 ( $23 \mathrm{~h}$ value $11 \%$ lower) together with a growth curve closely approximating to that observed for the Wood 46 strain. The 'high growth' clinical isolate no. 9 and the 'low growth' clinical isolate no. 10 (Fig. 1) produced relatively small amounts of exoprotein, apparently due to a premature cessation in synthesis compared with the other strains. In spite of the low yield of exoprotein it produced, even no. 10 appeared to exhibit an increase in the rate of exoprotein secretion after the end of exponential growth together with the biphasic differential rate of exoprotein formation which was characteristic of all other strains examined (Fig. 2).

\section{DISCUSSION}

The 10 random clinical isolates which showed widely differing exoprotein secretory abilities all have a property in common with the Wood 46 strain of $S$. aureus, namely, after the end of the exponential phase of growth the rate of exoprotein production increased. This finding is consistent with the idea of a regulatory mechanism based on 'competition' as suggested by Coleman et al. (1975) and discussed in relation to the Wood 46 strain of S. aureus by Abbas-Ali \& Coleman (1977) and Coleman \& Abbas-Ali (1977).

This characteristic, suggesting 'competition' between the cell protein and extracellular protein synthesizing machinery, was present, as nearly as one could tell within the limitations of the experimental technique available, even in the organism which showed the least ability to form exoproteins. However, this does not necessarily represent an inconsistency, since when discussing 'competition' we are considering a phenomenon which expresses itself during the exponential phase, when even those strains which ultimately produce the greatest amounts of exoprotein are not so readily distinguished from the others. Thus, for Wood 46 


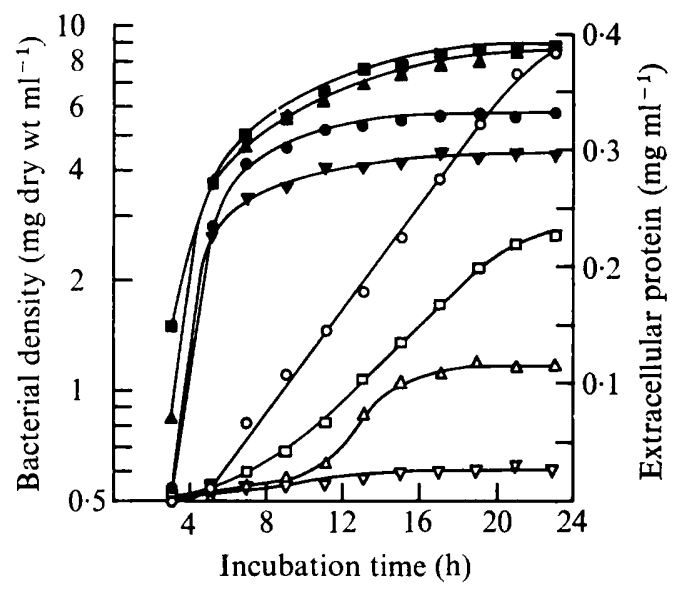

Fig. 1. Progress of extracellular protein secretion (open symbols) in relation to change in bacterial density (closed symbols) throughout the growth cycles of $S$. aureus Wood $46(\bigcirc, \bigcirc)$ and clinical isolates no. $7(\square, \square)$, no. $9(\triangle, \Delta)$ and no. $10(\nabla, \nabla)$.

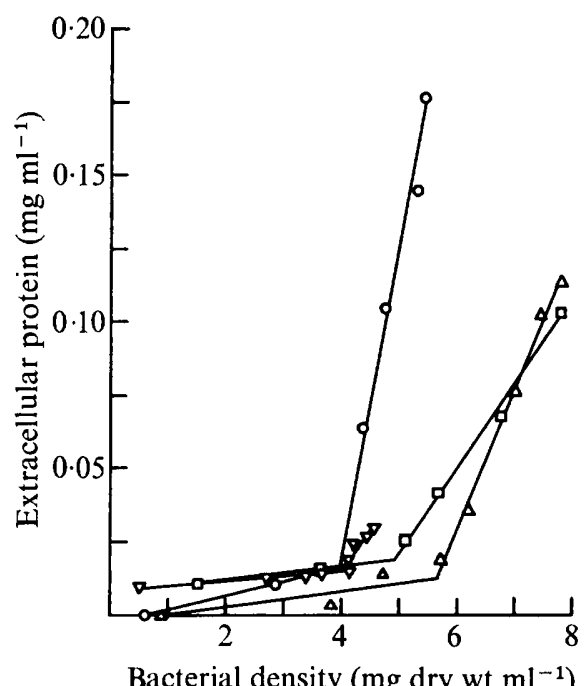

Bacterial density (mg dry wt $\mathrm{ml}^{-1}$ )

Fig. 2. Differential rates of extracellular protein formation by $S$. aureus Wood $46(O)$ and clinical isolates no. $7(\square)$, no. $9(\triangle)$ and no. $10(\nabla)$.

and clinical isolates no. 7 and no. 10, which achieved only $58 \%$ and $7 \%$ of the exoprotein level produced by Wood 46 over $23 \mathrm{~h}$, the differential rates of exoprotein synthesis in the exponential phase were $4 \cdot 5,2.5$, and $2.0 \mu \mathrm{g}$ exoprotein formed per $\mathrm{mg}$ dry wt increase in bacterial mass, respectively (Fig. 2).

The production of very high yields of exoprotein is a process which occurs after the end of exoponential growth. In clinical isolate no. 9, for example, limitation of exoprotein secretion resulted from a premature cessation of synthesis compared with the Wood 46 strain and clinical isolate no. 7 (Fig. 1). The reason for this 'switch off' of exoprotein formation is not known, but it may well have resulted from the loss of an essential component of the exoprotein synthesizing machinery.

The authors are indebted to Mrs Y. Thompson for her skilled technical assistance. 


\section{REFERENCES}

Abbas-Ali, B. \& Coleman, G. (1977). The characteristics of extracellular protein secretion by Staphylococcus aureus (Wood 46) and their relationship to the regulation of $\alpha$-toxin formation. Journal of General Microbiology 99, 277282.

BüRgi, W., Richterich, R. \& BRINeR, M. (1967). Ultraviolet photometric determination of total cerebrospinal fluid proteins with a modified biuret reagent. Clinica chimica acta 15, 181-184.

Coleman, G. \& Abbas-Ali, B. (1977). Comparison of the patterns of increase in $\alpha$-toxin and total extracellular protein by Staphylococcus aureus (Wood 46) grown in media supporting widely differing growth characteristics. Infection and Immunity 17, 278-281.

Coleman, G., Brown, S. \& Stormonth, D. A. (1975). A model for the regulation of bacterial extracellular enzyme and toxin biosynthesis. Journal of Theoretical Biology 52, 143-148.

Sedmak, J. J. \& Grossberg, S. E. (1977). A rapid, sensitive and versatile assay for protein using Coomassie brilliant blue G250. Analytical Biochemistry 79, 544-552.

Stormonth, D. A. \& Coleman, G. (1972). A rapid and convenient method for the determination of cell densities of bacteria which form aggregates. Journal of General Microbiology 71, 407-408. 